\title{
Quantum complex projective spaces from Toeplitz cubes
}

\author{
Piotr M. Hajac, Atabey Kaygun, and Bartosz Zieliński \\ Dedicated to Henri Moscovici on the occasion of his 65th birthday
}

\begin{abstract}
From $N$-tensor powers of the Toeplitz algebra, we construct a multi-pullback $\mathrm{C}^{*}$-algebra that is a noncommutative deformation of the complex projective space $\mathbb{P}^{N}(\mathbb{C})$. Using Birkhoff's Representation Theorem, we prove that the lattice of kernels of the canonical projections on components of the multi-pullback $\mathrm{C}^{*}$-algebra is free. This shows that our deformation preserves the freeness of the lattice of subsets generated by the affine covering of the complex projective space.
\end{abstract}

Mathematics Subject Classification (2010). 06B10, 06B25.

Keywords. Free distributive lattices, $\mathrm{C}^{*}$-ideals, multi-pullback $\mathrm{C}^{*}$-algebras.

\section{Introduction}

0.1. Motivation. The procedure of decomposing complicated spaces into the union of simple subsets and applying Mayer-Vietoris type arguments to understand thus decomposed spaces from the gluing data of simple pieces is commonly used in mathematics. Manifolds without boundary fit particularly well this piecewise approach because they are defined as spaces that are locally diffeomorphic to $\mathbb{R}^{n}$. A manifold is assembled from standard pieces by the gluing data. The standard pieces are contractible - they are homeomorphic to a ball. They encode only the dimension of a manifold. All the rest, topological properties of the manifold included, are described by the gluing data.

Recall that to study topological spaces, one typically uses open coverings. They are, however, hard to describe in purely $\mathrm{C}^{*}$-algebraic terms. On the other hand, the Gelfand transform turns closed coverings of a compact Hausdorff space $X$ into an appropriate set of surjections from the $\mathrm{C}^{*}$-algebra $C(X)$ of continuous functions on $X$ onto other $\mathrm{C}^{*}$-algebras. Hence it is easier and more natural to consider closed coverings if one wants a noncommutative generalisation in terms of $\mathrm{C}^{*}$-algebras. More specifically, one can define a covering of a quantum space to be a family of $\mathrm{C}^{*}$-algebra surjections whose kernels intersect to zero (cover the whole space). We refer to [14] and references therein for a more in-depth discussion of this issue. 
The aim of this article is to explore the method of constructing noncommutative deformations of manifolds by deforming the standard pieces. This method is an alternative to the global deformation methods. Thus it is expected to yield new examples or provide a new perspective on already known cases. Our deformation of the complex projective spaces is related to but different from a much studied quantumgroup example. (See the last section for details.) By construction, it is particularly suited for developing and testing a definition of the fiber-product of spectral triples that should describe a gluing of smooth (noncommutative) geometries along their boundaries.

Finally, let us note that complex projective spaces are topologically interesting manifolds equipped with non-trivial tautological line bundles. It seems very plausible that our Toeplitz projective spaces enjoy the same type of topological nontriviality and lead to interesting K-theoretic computations. They should also lead to non-crossed product U(1)-C*-algebras (non-trivial U(1) quantum principal bundles). Using index theory, this has already been achieved for $N=1$, i.e., for the mirror quantum sphere [16].

0.2. Main result. Our main result concerns a new noncommutative deformation of the complex projective space and the lattice generated by its affine covering. The guiding principle of our deformation is to preserve the gluing data of this manifold while deforming the standard pieces. We refine the affine covering of a complex projective space to the Cartesian powers of unit disks, and replace the algebra of continuous functions on the disk by the Toeplitz algebra commonly regarded as the algebra of a quantum disk [19]. The main point here is that we preserve the freeness property enjoyed by the lattice generated by the affine covering of the complex projective space:

Theorem 3.5. Let $C\left(\mathbb{P}^{N}(\mathcal{T})\right) \subset \prod_{i=0}^{N} \mathcal{T}^{\otimes N}$ be the $C^{*}$-algebra of the Toeplitz quantum projective space, and let $\pi_{i}: C\left(\mathbb{P}^{N}(\mathcal{T})\right) \rightarrow \mathcal{T}^{\otimes N}, i \in\{0, \ldots, N\}$, be the family of restrictions of the canonical projections onto the components. Then the family of ideals $\left\{\operatorname{ker} \pi_{i}\right\}_{i \in\{0, \ldots, N\}}$ generates a free distributive lattice.

0.3. Notation and conventions. In this article, the tensor product means the $\mathrm{C}^{*}$-completed tensor product. Accordingly, we use the Heynemann-Sweedler notation (with the summation sign suppressed) for the completed tensor product. Since all $\mathrm{C}^{*}$-algebras that we tensor are nuclear, this completion is unique. Therefore, it is also maximal, which guarantees the exactness of the completed tensor product. We use this property in our arguments. Since the subsets $\{0, \ldots, N\} \subset \mathbb{N}, N \in \mathbb{N}$, occur in abundance throughout this paper, for the sake brevity we use the notation

$$
\underline{N}:=\{0, \ldots, N\} .
$$




\section{Preliminaries}

1.1. Free lattices and Birkhoff's Representation Theorem. We first recall definitions and simple facts about ordered sets and lattices to fix terminology and notation. Our main references on the subject are [7], [8], [29].

A set $P$ together with a binary relation $\leqslant$ is called a partially ordered set, or a poset in short, if the relation $\leqslant$ is (i) reflexive, i.e., $p \leqslant p$ for any $p \in P$, (ii) transitive, i.e., $p \leqslant q$ and $q \leqslant r$ implies $p \leqslant r$ for any $p, q, r \in P$, and (iii) anti-symmetric, i.e., $p \leqslant q$ and $q \leqslant p$ implies $p=q$ for any $p, q \in P$. If only the conditions (i)-(ii) are satisfied we call $\leqslant$ a preorder. For every preordered set $(P, \leqslant)$ there is an opposite preordered set $(P, \leqslant)^{\text {op }}$ given by $P=P^{\text {op }}$ and $p \leqslant{ }^{\text {op }} q$ if and only if $q \leqslant p$ for any $p, q \in P$.

A poset $(P, \leqslant)$ is called a semi-lattice if for every $p, q \in P$ there exists an element $p \vee q$ such that (i) $p \leqslant p \vee q$, (ii) $q \leqslant p \vee q$, and (iii) if $r \in P$ is an element which satisfies $p \leqslant r$ and $q \leqslant r$ then $p \vee q \leqslant r$. The binary operation $\vee$ is called the join. A poset is called a lattice if both $(P, \leqslant)$ and $(P, \leqslant)^{\text {op }}$ are semi-lattices. The join operation in $P^{\text {op }}$ is called the meet, and traditionally denoted by $\wedge$. One can equivalently define a lattice $P$ as a set with two binary associative commutative and idempotent operations $\vee$ and $\wedge$. These operations satisfy two absorption laws: $p=p \vee(p \wedge q)$ and $p=p \wedge(p \vee q)$ for any $p, q \in P$. A lattice $(P, \vee, \wedge)$ is called distributive if one has $p \wedge(q \vee r)=(p \wedge q) \vee(p \wedge r)$ for any $p, q, r \in P$. Note that one can prove that the distributivity of meet over join we have here is equivalent to the distributivity of join over meet.

Let $(P, \leqslant)$ be a preordered set, and let $\uparrow p:=\{q \in P \mid p \leqslant q\}$ for any $p \in P$. As a natural extension of notation, we define $\uparrow U:=\bigcup_{p \in U} \uparrow p$ for any $U \subseteq P$. The subsets $U \subseteq P$ that satisfy $U=\uparrow U$ are called upper sets or dual order ideals.

Next, let $\Lambda$ be any lattice. An element $c \in \Lambda$ is called meet irreducible if

$$
\text { (i) } c=a \wedge b \Rightarrow(c=a \text { or } c=b) \text {, (ii) } \exists \lambda \in \Lambda: \lambda \not c \text {. }
$$

The set of meet irreducible elements of the lattice $\Lambda$ is denoted $\mathcal{M}(\Lambda)$. The join irreducibles $\mathcal{G}(\Lambda)$ are defined dually. Birkhoff's Representation Theorem [6] states that, if $\Lambda$ is a finite distributive lattice, then the map

$$
\Lambda \ni a \longmapsto\{x \in \mathcal{M}(\Lambda) \mid x \geqslant a\}=\mathcal{M}(\Lambda) \cap \uparrow a \in \operatorname{Up}(\mathcal{M}(\Lambda))
$$

assigning to $a$ the set of meet irreducible elements $\geqslant a$ is a lattice isomorphism between $\Lambda$ and the lattice $\operatorname{Up}(\mathcal{M}(\Lambda))$ of upper sets of meet-irreducible elements of $\Lambda$ with $\cap$ and $\cup$ as its join and meet, respectively. We refer to this isomorphism as the Birkhoff transform. Let us observe that it is analogous to the Gelfand transform: every finite distributive lattice is the lattice of upper sets of a certain poset just as every unital commutative $\mathrm{C}^{*}$-algebra is the algebra of continuous functions on a certain compact Hausdorff space.

As an immediate consequence of Birkhoff's Representation Theorem, one sees that two finite distributive lattices are isomorphic if and only if their posets of meet 
irreducibles are isomorphic. In particular, consider a free distributive lattice generated by $\lambda_{0}, \ldots, \lambda_{N}$, i.e., a lattice enjoying the universal property that it admits a lattice homomorphism into any distributive lattice generated by $N+1$ elements. It is isomorphic to the lattice of non-empty upper sets of the set of non-empty subsets of $\underline{N}$ (e.g., see [15], Sect. 2.2). The elements of the form $\bigvee_{i \in I} \lambda_{i}$, where $\emptyset \neq I \subsetneq \underline{N}$, are all meet irreducible and partially ordered by

$$
\bigvee_{i \in I} \lambda_{i} \leqslant \bigvee_{j \in J} \lambda_{j} \quad \text { if and only if } \quad I \subseteq J, \quad \forall I, J \neq \emptyset, I, J \subsetneq \underline{N} .
$$

Hence they are all distinct. Furthermore, all meet irreducible elements must be of the form $\bigvee_{i \in I} \lambda_{i}$, where $\emptyset \neq I \subsetneq \underline{N}$.

Note that the latter property holds for any finite distributive lattice. Indeed, suppose the contrary, i.e., that there exists a meet-irreducible element whose any presentation $\bigvee_{a \in \alpha} \bigwedge_{i \in a} \lambda_{i}$ is such that there is a set $a_{0} \in \alpha$ that contains at least two elements. Now, the finiteness allows us to apply induction, and the distributivity combined with irreducibility allows us to make the induction step yielding the desired contradiction.

Thus we conclude the following lemma:

Lemma 1.1. A finitely generated distributive lattice is free if the joins of its generators $\left\{\bigvee_{i \in I} \lambda_{i}\right\}_{\emptyset \neq I \subsetneq N}$ are all meet irreducible and satisfy (1).

1.2. Closed covering of $\mathbb{P}^{N}(\mathbb{C})$ as an example of a free lattice. In [15], a closed refinement of the affine covering of $\mathbb{P}^{N}(\mathbb{C})$ was constructed as an example of a finite closed covering of a compact Hausdorff space. Let us recall this construction. The elements of this covering are given by

$$
V_{i}:=\left\{\left[x_{0}: \ldots: x_{N}\right]|| x_{i} \mid=\max \left\{\left|x_{0}\right|, \ldots,\left|x_{N}\right|\right\}\right\}, \quad i \in \underline{N} .
$$

It is easy to see that the family $\left\{V_{i}\right\}_{i \in \underline{N}}$ of closed subsets of $\mathbb{P}^{N}(\mathbb{C})$ is a covering of $\mathbb{P}^{N}(\mathbb{C})$, i.e., $\bigcup_{i} V_{i}=\mathbb{P}^{N}(\mathbb{C})$. This covering is interesting because of its following property:

Proposition 1.2. The distributive lattice $\Lambda$ generated by the subsets $V_{i} \subset \mathbb{P}^{N}(\mathbb{C})$, $i \in \underline{N}$, is free.

Proof. We prove the freeness of $\Lambda$ by showing that $\Lambda$ is isomorphic as a lattice with the lattice $\Upsilon$ of non-empty upper sets of non-empty subsets of $\underline{N}$, which is a wellknown model of a free distributive lattice (see, e.g., [7]). For brevity, if $\emptyset \neq a \subseteq \underline{N}$, we write $V_{a}:=\bigcap_{i \in a} V_{i}$. Note that any $V \in \Lambda$ can be written as $V=\bigcup_{a \in A} V_{a}$ for some set $A$ of subsets of $\underline{N}$. We want to show that the following two maps are mutually inverse lattice isomorphisms:

$$
R: \Upsilon \ni X \longmapsto \bigcup_{a \in X} V_{a} \in \Lambda, \quad L: \Lambda \ni V \longmapsto\left\{a \in \underline{N} \mid V_{a} \subseteq V\right\} \in \Upsilon
$$


For a proof that $R$ is a lattice map see, e.g., [15], Sect. 2.2. The equality $R \circ L=\mathrm{id}$ is immediate.

The other equality $L \circ R=$ id can be proven as follows. Take any

$$
z_{a}:=\left[x_{0}: \ldots: x_{N}\right] \in \mathbb{P}^{N}(\mathbb{C}), \text { where }\left|x_{i}\right|=\max \left\{\left|x_{0}\right|, \ldots,\left|x_{N}\right|\right\} \Longleftrightarrow i \in a .
$$

Then one can easily see that $z_{a} \in V_{b} \Longleftrightarrow b \subseteq a$. Hence $z_{a} \in V \Longleftrightarrow V_{a} \subseteq V$, for all $V \in \Lambda$. Therefore, $a \in L(R(X))$ if and only if $z_{a} \in R(X)$, for all $X \in \Upsilon$. Finally, using again the property $z_{a} \in V_{b} \Longleftrightarrow b \subseteq a$ and the fact that $X$ is an upper set, we see that $z_{a} \in R(X)$ if and only if $a \in X$.

Now we use the covering $\left\{V_{i}\right\}_{i \in N}$ to present $\mathbb{P}^{N}(\mathbb{C})$ as a multi-pushout, and, consequently, its $\mathrm{C}^{*}$-algebra $C\left(\mathbb{P}^{N}(\overline{\mathbb{C}})\right)$ as a multi-pullback. To this end, we first define a family of homeomorphisms

$$
\begin{gathered}
\psi_{i}: V_{i} \longrightarrow D^{\times N}:=\underbrace{D \times \cdots \times D}_{N \text { times }}, \\
{\left[x_{0}: \ldots: x_{N}\right] \longmapsto\left(\frac{x_{0}}{x_{i}}, \ldots, \frac{x_{i-1}}{x_{i}}, \frac{x_{i+1}}{x_{i}}, \ldots, \frac{x_{N}}{x_{i}}\right),}
\end{gathered}
$$

for all $i \in \underline{N}$, from $V_{i}$ onto the Cartesian product of $N$-copies of the unit disk. The inverses of the maps $\psi_{i}$ are given explicitly by

$$
\psi_{i}^{-1}: D^{\times N} \ni\left(d_{1}, \ldots, d_{N}\right) \longmapsto\left[d_{1}: \ldots: d_{i}: 1: d_{i+1}: \ldots: d_{N}\right] \in \mathbb{P}^{N}(\mathbb{C}) .
$$

Pick indices $0 \leqslant i<j \leqslant N$ and consider the following commutative diagram:

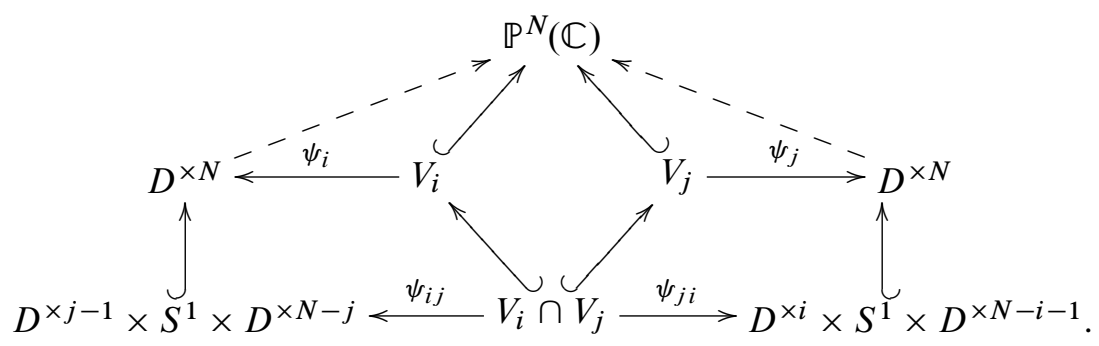

Here, for

$$
k= \begin{cases}n & \text { if } m<n \\ n+1 & \text { if } m>n\end{cases}
$$

we have

$$
\psi_{m n}:=\left.\psi_{m}\right|_{V_{m} \cap V_{n}}: V_{m} \cap V_{n} \longrightarrow D^{\times k-1} \times S^{1} \times D^{\times N-k} .
$$

In other words, counting from 1 , the unit circle $S^{1}$ appears on the $k$ th position among disks. It follows immediately from the definition of $\psi_{i}$ that the maps

$$
\Upsilon_{i j}:=\psi_{j i} \circ \psi_{i j}^{-1}: D^{\times j-1} \times S^{1} \times D^{\times N-j} \longrightarrow D^{\times i} \times S^{1} \times D^{\times N-i-1}, \quad i<j,
$$


can be explicitly written as

$$
\begin{aligned}
& \Upsilon_{i j}\left(d_{1}, \ldots, d_{j-1}, s, d_{j+1}, \ldots, d_{N}\right) \\
& \quad=\left(s^{-1} d_{1}, \ldots, s^{-1} d_{i}, s^{-1}, s^{-1} d_{i+1}, \ldots, s^{-1} d_{j-1}, s^{-1} d_{j+1}, \ldots, s^{-1} d_{N}\right)
\end{aligned}
$$

One can see from the diagram (2) that $\mathbb{P}^{N}(\mathbb{C})$ is homeomorphic to the disjoint union $\bigsqcup_{i=0}^{N} D_{i}^{\times N}$ of $(N+1)$-copies of $D^{\times N}$ divided by the identifications prescribed by the following diagrams indexed by $i, j \in \underline{N}, i<j$,

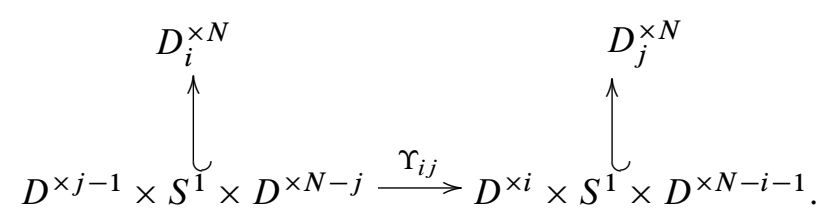

Consequently, one sees that the $\mathrm{C}^{*}$-algebra $C\left(\mathbb{P}^{N}(\mathbb{C})\right)$ of continuous functions on $\mathbb{P}^{N}(\mathbb{C})$ is isomorphic with the subalgebra of $\prod_{i=0}^{N} C(D)_{i}^{\otimes N}$ defined by the compatibility conditions given by the diagrams dual to the diagrams (4):

$$
\begin{gathered}
C(D)_{i}^{\otimes N} \\
\Downarrow \\
\qquad(D)^{\otimes j-1} \otimes C\left(S^{1}\right) \otimes C(D)^{\times N-j} \stackrel{\Upsilon_{i j}^{*}}{\leftarrow} C(D)^{\otimes i} \otimes C\left(S^{1}\right) \otimes C(D)^{\otimes N-i-1} .
\end{gathered}
$$

\section{The multi-pullback $C^{*}$-algebra of $\mathbb{P}^{N}(\mathcal{T})$}

As a starting point for our noncommutative deformation of a complex projective space, we take the diagrams (5) from Section 1.2 and replace the algebra $C(D)$ of continuous functions on the unit disk by the Toeplitz algebra $\mathcal{T}$ considered as the algebra of continuous functions on a quantum disk [19]. Recall that the Toeplitz algebra is the universal $C^{*}$-algebra generated by $z$ and $z^{*}$ satisfying $z^{*} z=1$. There is a well-known short exact sequence of $\mathrm{C}^{*}$-algebras

$$
0 \longrightarrow \mathcal{K} \longrightarrow \mathcal{T} \stackrel{\sigma}{\longrightarrow} C\left(S^{1}\right) \longrightarrow 0
$$

Here $\sigma$ is the so-called symbol map defined by mapping $z$ to the unitary generator $u$ of the algebra $C\left(S^{1}\right)$ of continuous functions on a circle. Note that the kernel of the symbol map is the algebra $\mathcal{K}$ of compact operators.

Viewing $S^{1}$ as the unitary group $\mathrm{U}(1)$, we obtain a compact quantum group structure on the algebra $C\left(S^{1}\right)$. Here the antipode is determined by $S(u)=u^{-1}$, the counit by $\varepsilon(u)=1$, and finally the comultiplication by $\Delta(u)=u \otimes u$. The 
Hopf-algebraic structure exists on the $\mathrm{C}^{*}$-level due to the commutativity of $C\left(S^{1}\right)$. The coaction of $C\left(S^{1}\right)$ on $\mathcal{T}$ comes from the gauge action of $U(1)$ on $\mathcal{T}$ that rescales $z$ by the elements of $\mathrm{U}(1)$, i.e., $z \mapsto \lambda z$. Explicitly, we have:

$\rho: \mathcal{T} \rightarrow \mathcal{T} \otimes C\left(S^{1}\right) \cong C\left(S^{1}, \mathcal{T}\right), \rho(z):=z \otimes u, \rho(z)(\lambda)=\lambda z, \rho(t)=: t^{(0)} \otimes t^{(1)}$.

Next, we employ the multiplication map $m$ of $C\left(S^{1}\right)$ and the flip map

$$
C\left(S^{1}\right) \otimes \mathcal{T}^{\otimes n} \ni f \otimes t_{1} \otimes \cdots \otimes t_{n} \stackrel{\tau_{n}}{\longmapsto} t_{1} \otimes \cdots \otimes t_{n} \otimes f \in \mathcal{T}^{\otimes n} \otimes C\left(S^{1}\right)
$$

to extend $\rho$ to the diagonal coaction $\rho_{n}: \mathcal{T}^{\otimes n} \longrightarrow \mathcal{T}^{\otimes n} \otimes C\left(S^{1}\right)$ defined inductively by

$$
\rho_{1}=\rho, \quad \rho_{n+1}=\left(\operatorname{id}_{\mathcal{T} \otimes n+1} \otimes m\right) \circ\left(\operatorname{id}_{\mathcal{T}} \otimes \tau_{n} \otimes \operatorname{id}_{C\left(S^{1}\right)}\right) \circ\left(\rho \otimes \rho_{n}\right) .
$$

Furthermore, for all $0 \leqslant i<j \leqslant N$, we define an isomorphism $\Psi_{i j}$ by

$$
\chi_{j} \circ \Psi \circ \chi_{i+1}^{-1}: \mathcal{T}^{\otimes i} \otimes C\left(S^{1}\right) \otimes \mathcal{T}^{\otimes N-i-1} \stackrel{\Psi_{i j}}{\longrightarrow} \mathcal{T}^{\otimes j-1} \otimes C\left(S^{1}\right) \otimes \mathcal{T}^{\otimes N-j} .
$$

Here $\chi_{j}$ is given by

$$
\mathrm{id}_{\mathcal{T} \otimes j-1} \otimes \tau_{N-j}^{-1}: \mathcal{T}^{\otimes N-1} \otimes C\left(S^{1}\right) \stackrel{\chi_{j}}{\longrightarrow} \mathcal{T}^{\otimes j-1} \otimes C\left(S^{1}\right) \otimes \mathcal{T}^{\otimes N-j}
$$

and $\Psi$ by

$\left(\operatorname{id}_{\mathcal{T} \otimes N-1} \otimes(S \circ m)\right) \circ\left(\rho_{N-1} \otimes \operatorname{id}_{C\left(S^{1}\right)}\right): \mathcal{T}^{\otimes N-1} \otimes C\left(S^{1}\right) \stackrel{\Psi}{\rightarrow} \mathcal{T}^{\otimes N-1} \otimes C\left(S^{1}\right)$.

Before proceeding further, let us prove the unipotent property of $\Psi$, which we shall need later on.

Lemma 2.1. $\Psi \circ \Psi=\mathrm{id}_{\mathcal{T} \otimes N-1 \otimes C\left(S^{1}\right)}$.

Proof. For any $\bigotimes_{1 \leqslant i<N} t_{i} \otimes h \in \mathcal{T}^{\otimes N} \otimes C\left(S^{1}\right)$, we compute:

$$
\begin{aligned}
(\Psi \circ \Psi)\left(\bigotimes_{1 \leqslant i<N} t_{i} \otimes h\right) & =\Psi\left(\bigotimes_{1 \leqslant i<N} t_{i}^{(0)} \otimes S\left(\prod_{1 \leqslant i<N} t_{i}^{(1)} h\right)\right) \\
& =\bigotimes_{1 \leqslant i<N} t_{i}^{(0)} \otimes S\left(\left(\prod_{1 \leqslant i<N} t_{i}^{(1)}\right) S\left(\prod_{1 \leqslant j<N} t_{j}^{(2)} h\right)\right) \\
& =\bigotimes_{1 \leqslant i<N} t_{i}^{(0)} \otimes S\left(\left(\prod_{1 \leqslant i<N}\left(t_{i}^{(1)}\right) S\left(t_{i}^{(2)}\right)\right) S(h)\right) \\
& =\bigotimes_{1 \leqslant i<N} t_{i} \otimes h .
\end{aligned}
$$

Finally, to justify our construction of a quantum complex projective space, observe that the map $\Psi_{i j}$ can be easily seen as an analogue of the pullback of the map $\Upsilon_{i j}$ of (3). 
Definition 2.2. We define the $\mathrm{C}^{*}$-algebra $C\left(\mathbb{P}^{N}(\mathcal{T})\right)$ as the limit of the diagram:

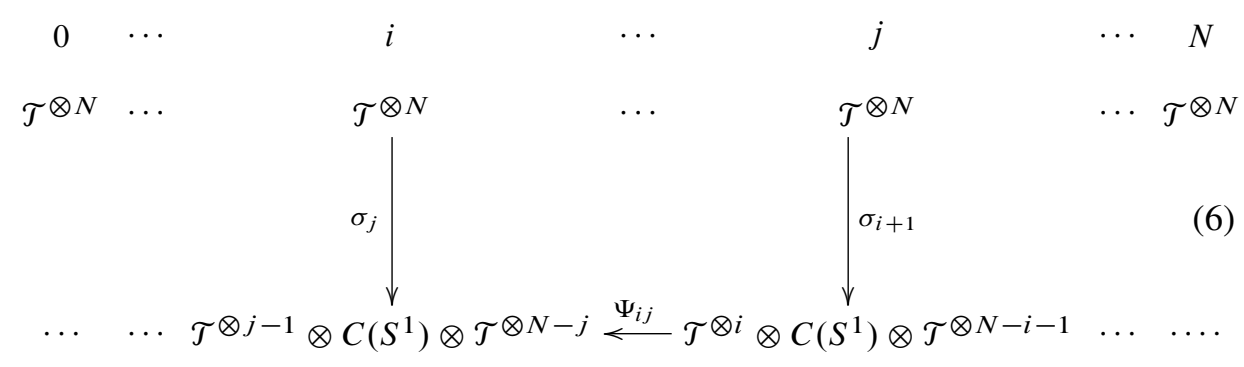

Here we take all $i, j \in \underline{N}, i<j$, and define $\sigma_{k}:=\mathrm{id}_{\mathcal{T} \otimes k-1} \otimes \sigma \otimes \mathrm{id}_{\mathcal{T} \otimes N-k}$, $k \in\{1, \ldots, N\}$. We call $\mathbb{P}^{N}(\mathcal{T})$ a Toeplitz quantum complex projective space.

Note that by definition $C\left(\mathbb{P}^{N}(\mathcal{T})\right) \subseteq \prod_{i=0}^{N} \mathcal{T}^{\otimes N}$. We will denote the restrictions of the canonical projections on the components by

$$
\pi_{i}: C\left(\mathbb{P}^{N}(\mathcal{T})\right) \longrightarrow \mathcal{T}^{\otimes N}, \quad \forall i \in \underline{N} .
$$

Since these maps are $\mathrm{C}^{*}$-homomorphisms, the lattice generated by their kernels is automatically distributive. On the other hand, it follows from Lemma 3.2 that any element in the Toeplitz cube $\mathcal{T}^{\otimes n}$ can be complemented into a sequence that is an element of $C\left(\mathbb{P}^{N}(\mathcal{T})\right)$. This means that the maps (7) are surjective. Hence they form a covering of $C\left(\mathbb{P}^{N}(\mathcal{T})\right)$.

The construction of $\mathbb{P}^{N}(\mathcal{T})$ is a generalization of the construction of the mirror quantum sphere [16], p. 734 , i.e., $\mathbb{P}^{1}(\mathcal{T})$ is the mirror quantum sphere:

$$
C\left(\mathbb{P}^{1}(\mathcal{T})\right):=\left\{\left(t_{0}, t_{1}\right) \in \mathcal{T} \times \mathcal{T} \mid \sigma\left(t_{0}\right)=S\left(\sigma\left(t_{1}\right)\right)\right\} .
$$

Removing $S$ from this definition yields the $\mathrm{C}^{*}$-algebra of the generic Podleś sphere [23]. The latter not only is not isomorphic with $C\left(\mathbb{P}^{1}(\mathcal{T})\right)$ but also is not Morita equivalent to $C\left(\mathbb{P}^{1}(\mathcal{T})\right)$ [16], Prop. 2.3. We conjecture that, by similar changes in maps $\Psi_{i j}$, we can create non-equivalent quantum spaces also for $N>1$.

\section{The defining covering lattice of $\mathbb{P}^{N}(\mathcal{T})$ is free}

The goal of this section is to demonstrate that the distributive lattice of ideals generated by the kernels $\operatorname{ker} \pi_{i}$ is free. To this end, we will need to know whether the tensor products $\mathcal{T}^{\otimes N}$ of Toeplitz algebras glue together to form $\mathbb{P}^{N}(\mathcal{T})$ in such a way that a partial gluing can always be extended to the full space. The following result gives sufficient conditions: 
Proposition 3.1 ([9], Prop. 9). Let $\left\{B_{i}\right\}_{i \in \underline{N}}$ and $\left\{B_{i j}\right\}_{i, j \in \underline{N}, i \neq j}$ be two families of $C^{*}$-algebras such that $B_{i j}=B_{j i}$, and let $\left\{\pi_{j}^{i}: B_{i} \rightarrow B_{i j}\right\}_{i j}$ be a family of surjective $C^{*}$-algebra maps. Also, let $\pi_{i}: B \rightarrow B_{i}, i \in \underline{N}$, be the restrictions to

$$
B:=\left\{\left(b_{i}\right)_{i} \in \prod_{i \in \underline{N}} B_{i} \mid \pi_{j}^{i}\left(b_{i}\right)=\pi_{i}^{j}\left(b_{j}\right), \forall i, j \in \underline{N}, i \neq j\right\}
$$

of the canonical projections. Assume that, for all triples of distinct indices $i, j, k \in \underline{N}$,

(1) $\pi_{j}^{i}\left(\operatorname{ker} \pi_{k}^{i}\right)=\pi_{i}^{j}\left(\operatorname{ker} \pi_{k}^{j}\right)$,

(2) the isomorphisms $\pi_{k}^{i j}: B_{i} /\left(\operatorname{ker} \pi_{j}^{i}+\operatorname{ker} \pi_{k}^{i}\right) \longrightarrow B_{i j} / \pi_{j}^{i}\left(\operatorname{ker} \pi_{k}^{i}\right)$ defined as

$$
b_{i}+\operatorname{ker} \pi_{j}^{i}+\operatorname{ker} \pi_{k}^{i} \longmapsto \pi_{j}^{i}\left(b_{i}\right)+\pi_{j}^{i}\left(\operatorname{ker} \pi_{k}^{i}\right)
$$

satisfy

$$
\left(\pi_{j}^{i k}\right)^{-1} \circ \pi_{j}^{k i}=\left(\pi_{k}^{i j}\right)^{-1} \circ \pi_{k}^{j i} \circ\left(\pi_{i}^{j k}\right)^{-1} \circ \pi_{i}^{k j}
$$

Then,

$$
\begin{gathered}
\forall\left(b_{i}\right)_{i \in I} \in \prod_{i \in I} B_{i}, I \subseteq \underline{N}, \text { such that } \pi_{j}^{i}\left(b_{i}\right)=\pi_{i}^{j}\left(b_{j}\right), \forall i, j \in I, i \neq j, \\
\exists\left(c_{i}\right)_{i \in \underline{N}} \in \prod_{i \in \underline{N}} B_{i}: \pi_{j}^{i}\left(c_{i}\right)=\pi_{i}^{j}\left(c_{j}\right), \forall i, j \in \underline{N}, i \neq j, \text { and } c_{i}=b_{i}, \forall i \in I .
\end{gathered}
$$

In the case of quantum projective spaces $\mathbb{P}^{N}(\mathcal{T})$, we can translate algebras and maps from Proposition 3.1 as follows:

$$
\begin{aligned}
B_{i}=\mathcal{T}^{\otimes N}, \quad & B_{i j}=\mathcal{T}^{\otimes j-1} \otimes C\left(S^{1}\right) \otimes \mathcal{T}^{\otimes N-j}, \text { where } i<j, \\
\pi_{j}^{i} & = \begin{cases}\sigma_{j} & \text { when } i<j, \\
\Psi_{j i} \circ \sigma_{j+1} & \text { when } i>j .\end{cases}
\end{aligned}
$$

It follows that

$$
\operatorname{ker} \pi_{j}^{i}= \begin{cases}\operatorname{ker} \sigma_{j}=\mathcal{T}^{\otimes j-1} \otimes \mathcal{K} \otimes \mathcal{T}^{\otimes N-j} & \text { when } i<j, \\ \operatorname{ker} \sigma_{j+1}=\mathcal{T}^{\otimes j} \otimes \mathcal{K} \otimes \mathcal{T}^{\otimes N-j-1} & \text { when } i>j\end{cases}
$$

Since $\rho(\mathcal{K}) \subseteq \mathcal{K} \otimes C\left(S^{1}\right)$ and $\Psi$ is an isomorphism by Lemma 2.1, it follows that $\Psi\left(\mathcal{T}^{\otimes j-1} \otimes \mathcal{K} \otimes \mathcal{T}^{\otimes N-j-1} \otimes C\left(S^{1}\right)\right)=\mathcal{T}^{\otimes j-1} \otimes \mathcal{K} \otimes \mathcal{T}^{\otimes N-j-1} \otimes C\left(S^{1}\right)$.

Now we can formulate and prove the following:

Lemma 3.2. If $\left(b_{i}\right)_{i \in I} \in \prod_{i \in I \subseteq \underline{N}} \mathcal{T}^{\otimes N}$ satisfies $\pi_{j}^{i}\left(b_{i}\right)=\pi_{i}^{j}\left(b_{j}\right)$ for all $i, j \in I$, $i \neq j$, then there exists an element $b \in C\left(\mathbb{P}^{N}(\mathcal{T})\right)$ such that $\pi_{i}(b)=b_{i}$ for all $i \in I$. 
Proof. It is enough to check that the assumptions of Proposition 3.1 are satisfied. For the sake of brevity, we will omit the tensor symbols in the long formulas in what follows. We will also write $S$ instead of $C\left(S^{1}\right)$. Here we prove the first condition of Proposition 3.1:

(1) $\pi_{i}^{j}\left(\operatorname{ker} \pi_{k}^{j}\right)=\left(\chi_{j} \circ \Psi \circ \chi_{i+1}^{-1} \circ \sigma_{i+1}\right)\left(\operatorname{ker} \sigma_{k+1}\right)$

$$
\begin{aligned}
& =\left(\chi_{j} \circ \Psi \circ \chi_{i+1}^{-1}\right)\left(\mathcal{T}^{i} \delta \mathcal{T}^{k-i-1} \mathcal{K} \mathcal{T}^{N-k-1}\right) \\
& =\chi_{j}\left(\mathcal{T}^{k-1} \mathcal{K} \mathcal{T}^{N-k-1} \S\right) \\
& =\mathcal{T}^{k-1} \mathcal{K} \mathcal{T}^{j-k-1} \delta \mathcal{T}^{N-j} \\
& =\sigma_{j}\left(\operatorname{ker} \sigma_{k}\right) \\
& =\pi_{j}^{i}\left(\operatorname{ker} \pi_{k}^{i}\right), \text { when } i<k<j .
\end{aligned}
$$

(2) $\pi_{i}^{j}\left(\operatorname{ker} \pi_{k}^{j}\right)=\left(\chi_{j} \circ \Psi \circ \chi_{i+1}^{-1} \circ \sigma_{i+1}\right)\left(\operatorname{ker} \sigma_{k}\right)$

$$
\begin{aligned}
& =\left(\chi_{j} \circ \Psi \circ \chi_{i+1}^{-1}\right)\left(\mathcal{T}^{i} \delta \mathcal{T}^{k-i-2} \mathcal{K} \mathcal{T}^{N-k}\right) \\
& =\chi_{j}\left(\mathcal{T}^{k-2} \mathcal{K} \mathcal{T}^{N-k} \S\right) \\
& =\mathcal{T}^{j-1} \delta \mathcal{T}^{k-j-1} \mathcal{K} \mathcal{T}^{N-k} \\
& =\sigma_{j}\left(\operatorname{ker} \sigma_{k}\right) \\
& =\pi_{j}^{i}\left(\operatorname{ker} \pi_{k}^{i}\right), \text { when } i<j<k .
\end{aligned}
$$

(3) $\pi_{i}^{j}\left(\operatorname{ker} \pi_{k}^{j}\right)=\left(\chi_{j} \circ \Psi \circ \chi_{i+1}^{-1} \circ \sigma_{i+1}\right)\left(\operatorname{ker} \sigma_{k+1}\right)$

$$
\begin{aligned}
& =\left(\chi_{j} \circ \Psi \circ \chi_{i+1}^{-1}\right)\left(\mathcal{T}^{k} \mathcal{K} \mathcal{T}^{i-k-1} \delta \mathcal{T}^{N-i-1}\right) \\
& =\chi_{j}\left(\mathcal{T}^{k} \mathcal{K} \mathcal{T}^{N-k-2} \mathcal{S}\right) \\
& =\mathcal{T}^{k} \mathcal{K} \mathcal{T}^{j-k-2} \delta \mathcal{T}^{N-j} \\
& =\sigma_{j}\left(\operatorname{ker} \sigma_{k+1}\right) \\
& =\pi_{j}^{i}\left(\operatorname{ker} \pi_{k}^{i}\right), \text { when } k<i<j .
\end{aligned}
$$

For the second condition, note first that for any multi-valued map $f: B_{j} \rightarrow B_{i}$ we can define the map

$$
\begin{aligned}
{[f]_{k}^{i j}: } & B_{j} /\left(\operatorname{ker} \pi_{i}^{j}+\operatorname{ker} \pi_{k}^{j}\right) \longrightarrow B_{i} /\left(\operatorname{ker} \pi_{j}^{i}+\operatorname{ker} \pi_{k}^{i}\right), \\
b_{j}+\operatorname{ker} \pi_{i}^{j}+\operatorname{ker} \pi_{k}^{j} & \longmapsto f\left(b_{j}\right)+\operatorname{ker} \pi_{j}^{i}+\operatorname{ker} \pi_{k}^{i},
\end{aligned}
$$

whenever the assignment (8) is unique. In particular, since the condition (1) of Proposition 3.1 is fulfilled, the map $\phi_{k}^{i j}:=\left(\pi_{k}^{i j}\right)^{-1} \circ \pi_{k}^{j i}$ exists, and we can write it as $\left[\left(\pi_{j}^{i}\right)^{-1} \circ \pi_{i}^{j}\right]_{k}^{i j}$. Explicitly, in our case, this map reads:

$$
\phi_{k}^{i j}= \begin{cases}{\left[\sigma_{j}^{-1} \circ \chi_{j} \circ \Psi \circ \chi_{i+1}^{-1} \circ \sigma_{i+1}\right]_{k}^{i j}} & \text { when } i<j, \\ {\left[\sigma_{j+1}^{-1} \circ \chi_{j+1} \circ \Psi \circ \chi_{i}^{-1} \circ \sigma_{i}\right]_{k}^{i j}} & \text { when } i>j .\end{cases}
$$


We need to prove that

$$
\phi_{k}^{i j}=\phi_{j}^{i k} \circ \phi_{i}^{k j}, \quad \text { for all distinct indices } i, j, k \text {. }
$$

Since $\left(\phi_{k}^{i j}\right)^{-1}=\phi_{k}^{j i}$ and, for any invertible elements $g, h, k$, the equality $k=g h$ can be written as $h=g^{-1} k$, etc., one can readily see that it is enough to limit ourselves to the case when $i<k<j$. Next, let us denote the class of $\left(t_{1} \otimes \cdots \otimes t_{N}\right)=$ $\bigotimes_{1 \leqslant n \leqslant N} t_{n} \in \mathcal{T}^{\otimes N}$ in $\mathcal{T}^{\otimes N} /\left(\operatorname{ker} \pi_{i}^{j}+\operatorname{ker} \pi_{k}^{j}\right)$ by $\left[\bigotimes_{1 \leqslant n \leqslant N} t_{n}\right]_{i k}^{j}$. Then, using the Heynemann-Sweedler notation for completed tensor products, we compute

$$
\begin{aligned}
\phi_{k}^{i j}\left(\left[\bigotimes_{1 \leqslant n \leqslant N} t_{n}\right]_{i k}^{j}\right) & =\left[\sigma_{j}^{-1} \circ \chi_{j} \circ \Psi \circ \chi_{i+1}^{-1} \circ \sigma_{i+1}\right]_{k}^{i j}\left(\left[\bigotimes_{1 \leqslant n \leqslant N}^{\otimes} t_{n}\right]_{i k}^{j}\right) \\
& =\left[\left(\sigma_{j}^{-1} \circ \chi_{j} \circ \Psi\right)\left(\bigotimes_{\substack{1 \leqslant n \leqslant N \\
n \neq i+1}}^{\bigotimes} t_{n} \otimes \sigma\left(t_{i+1}\right)\right)\right]_{j k}^{i} \\
& =\left[\left(\sigma_{j}^{-1} \circ \chi_{j}\right)\left(\bigotimes_{\substack{1 \leqslant n \leqslant N \\
n \neq i+1}}^{(0)} t_{n}^{(0)} \otimes S\left(\sigma\left(t_{i+1}\right) \prod_{\substack{1 \leqslant m \leqslant N \\
m \neq i+1}} t_{m}^{(1)}\right)\right)\right]_{j k}^{i} \\
& =\left[\underset{\substack{1 \leqslant n \leqslant j \\
n \neq i+1}}{\otimes} t_{n}^{(0)} \otimes\left(\sigma^{-1} \circ S\right)\left(\sigma\left(t_{i+1}\right) \prod_{\substack{1 \leqslant m \leqslant N \\
m \neq i+1}}^{(1)} t_{m} \otimes \bigotimes_{j+1 \leqslant s \leqslant N}^{\otimes} t_{s}^{(0)}\right]_{j k}^{i} .\right.
\end{aligned}
$$

Applying the above formula twice (with the non-dummy indices changed), we obtain

$$
\begin{aligned}
& \left(\phi_{j}^{i k} \circ \phi_{i}^{k j}\right)\left(\left[\bigotimes_{1 \leqslant n \leqslant N} t_{n}\right]_{i k}^{j}\right) \\
& =\phi_{j}^{i k}\left(\left[\bigotimes_{\substack{1 \leqslant n \leqslant j \\
n \neq k+1}} t_{n}^{(0)} \otimes\left(\sigma^{-1} \circ S\right)\left(\sigma\left(t_{k+1}\right) \prod_{\substack{1 \leqslant m \leqslant N \\
m \neq k+1}} t_{m}^{(1)}\right) \otimes \bigotimes_{j+1 \leqslant s \leqslant N} t_{s}^{(0)}\right]_{j i}^{k}\right) \\
& =\left[\bigotimes_{\substack{1 \leqslant n \leqslant k \\
n \neq i+1}} t_{n}^{(0)(0)} \otimes\left(\sigma^{-1} \circ S\right)\left(\sigma\left(t_{i+1}^{(0)}\right)\left(\left(\sigma^{-1} \circ S\right)\left(\sigma\left(t_{k+1}\right) \prod_{\substack{1 \leqslant m \leqslant N \\
m \neq k+1}} t_{m}^{(1)}\right)\right)^{(1)} \prod_{\substack{1 \leqslant w \leqslant N \\
w \neq i+1 \\
w \neq k+1}} t_{w}^{(0)(1)}\right)\right. \\
& \otimes \bigotimes_{k+2 \leqslant r \leqslant j} t_{n}^{(0)(0)} \otimes\left(\left(\sigma^{-1} \circ S\right)\left(\sigma\left(t_{k+1}\right) \prod_{\substack{1 \leqslant m \leqslant N \\
m \neq k+1}}^{(1)} t_{m}^{(0)} \otimes \bigotimes_{j+1 \leqslant s \leqslant N} t_{s}^{(0)(0)}\right]_{j k}^{i} .\right.
\end{aligned}
$$

Now, as $\sigma^{-1}: C\left(S^{1}\right) \rightarrow \mathcal{T} / \mathcal{K}$ is colinear, $S$ is an anti-coalgebra map, and $\Delta$ is an algebra homomorphism, we can move the Heynemann-Sweedler indices inside the bold parentheses:

$$
\begin{aligned}
& {\left[\bigotimes_{\substack{1 \leqslant n \leqslant k \\
n \neq i+1}} t_{n}^{(0)(0)} \otimes\left(\sigma^{-1} \circ S\right)\left(\sigma\left(t_{i+1}^{(0)}\right) S\left(\sigma\left(t_{k+1}\right)^{(1)} \prod_{\substack{1 \leqslant m \leqslant N \\
m \neq k+1}} t_{m}^{(1)(1)}\right) \prod_{\substack{1 \leqslant w \leqslant N \\
w \neq i+1 \\
w \neq k+1}} t_{w}^{(0)(1)}\right)\right.} \\
& \left.\otimes \bigotimes_{k+2 \leqslant r \leqslant j} t_{n}^{(0)(0)} \otimes\left(\sigma^{-1} \circ S\right)\left(\sigma\left(t_{k+1}\right)^{(2)} \prod_{\substack{1 \leqslant m \leqslant N \\
m \neq k+1}} t_{m}^{(1)(2)}\right) \otimes \bigotimes_{j+1 \leqslant s \leqslant N} t_{s}^{(0)(0)}\right]_{j k}^{i} .
\end{aligned}
$$

Here we can renumber the Heynemann-Sweedler indices using the coassociativity of $\Delta$. We can also use the anti-multiplicativity of $S$ to move it inside the bold 
parentheses in the first line of the above calculation. Finally, we use the commutativity of $C\left(S^{1}\right)$ in order to reshuffle the argument of $\sigma^{-1} \circ S$ in the first line to obtain

$$
\begin{aligned}
& {\left[\bigotimes_{\substack{1 \leqslant n \leqslant k \\
n \neq i+1}} t_{n}^{(0)} \otimes\left(\sigma^{-1} \circ S\right)\left(\sigma\left(t_{i+1}^{(0)}\right) S\left(t_{i+1}^{(1)}\right) S\left(\sigma\left(t_{k+1}\right)^{(1)}\right) \prod_{\substack{1 \leqslant w \leqslant N \\
w \neq i+1 \\
w \neq k+1}}\left(t_{w}^{(1)} S\left(t_{w}^{(2)}\right)\right)\right)\right.} \\
& \left.\quad \otimes \bigotimes_{k+2 \leqslant r \leqslant j} t_{n}^{(0)} \otimes\left(\sigma^{-1} \circ S\right)\left(\sigma\left(t_{k+1}\right)^{(2)} t_{i+1}^{(2)} \prod_{\substack{1 \leqslant m \leqslant N \\
m \neq k+1 \\
m \neq i+1}} t_{m}^{(3)}\right) \otimes \bigotimes_{j+1 \leqslant s \leqslant N} t_{s}^{(0)}\right]_{j k}^{i}
\end{aligned}
$$

We can simplify the expression in the bold parentheses in the first line using $h^{(1)} S\left(h^{(2)}\right)=\varepsilon(h)$ and $\varepsilon\left(h^{(1)}\right) h^{(2)}=h$. This results in

$$
\begin{aligned}
& {\left[\bigotimes_{\substack{1 \leq n \leq k \\
n \neq i+1}} t_{n}^{(0)} \otimes\left(\sigma^{-1} \circ S\right)\left(\sigma\left(t_{i+1}^{(0)}\right) S\left(t_{i+1}^{(1)}\right) S\left(\sigma\left(t_{k+1}\right)^{(1)}\right)\right)\right.} \\
& \left.\quad \otimes \bigotimes_{\substack{k+2 \leqslant r \leqslant j \\
t_{n}}} t_{n}^{(0)} \otimes\left(\sigma^{-1} \circ S\right)\left(\sigma\left(t_{k+1}\right)^{(2)} t_{i+1}^{(2)} \prod_{\substack{1 \leqslant m \leqslant N \\
m \neq k+1 \\
m \neq i+1}} t_{m}^{(1)}\right) \otimes \bigotimes_{j+1 \leqslant s \leqslant N} t_{s}^{(0)}\right]_{j k}^{i} .
\end{aligned}
$$

By the colinearity of $\sigma$, we can substitute in the above expression

$$
\begin{aligned}
\sigma\left(t_{i+1}^{(0)}\right) \otimes t_{i+1}^{(1)} & \mapsto \sigma\left(t_{i+1}\right)^{(1)} \otimes \sigma\left(t_{i+1}\right)^{(2)}, \\
\sigma\left(t_{k+1}\right)^{(1)} \otimes \sigma\left(t_{k+1}\right)^{(2)} & \mapsto \sigma\left(t_{k+1}^{(0)}\right) \otimes t_{k+1}^{(1)},
\end{aligned}
$$

to derive

$$
\begin{aligned}
& {\left[\bigotimes_{\substack{1 \leqslant n \leqslant k \\
n \neq i+1}} t_{n}^{(0)} \otimes\left(\sigma^{-1} \circ S\right)\left(\sigma\left(t_{i+1}\right)^{(1)} S\left(\sigma\left(t_{i+1}\right)^{(2)}\right) S\left(\sigma\left(t_{k+1}^{(0)}\right)\right)\right)\right.} \\
& \left.\quad \otimes \bigotimes_{k+2 \leqslant r \leqslant j} t_{n}^{(0)} \otimes\left(\sigma^{-1} \circ S\right)\left(t_{k+1}^{(1)} \sigma\left(t_{i+1}\right)^{(3)} \prod_{\substack{1 \leq m \leq N \\
m \neq k+1 \\
m \neq i+1}} t_{m}^{(1)}\right) \otimes \bigotimes_{j+1 \leqslant s \leqslant N} t_{s}^{(0)}\right]_{j k}^{i} .
\end{aligned}
$$

Applying again the antipode and counit properties yields the desired:

$$
\begin{aligned}
& {\left[\bigotimes_{\substack{1 \leqslant n \leqslant k \\
n \neq i+1}} t_{n}^{(0)} \otimes\left(\sigma^{-1} \circ S\right)\left(S\left(\sigma\left(t_{k+1}^{(0)}\right)\right)\right)\right.} \\
& \left.\quad \otimes \bigotimes_{k+2 \leqslant r \leqslant j} t_{n}^{(0)} \otimes\left(\sigma^{-1} \circ S\right)\left(t_{k+1}^{(1)} \sigma\left(t_{i+1}\right) \prod_{\substack{1 \leq m \leq N \\
m \neq k+1 \\
m \neq i+1}} t_{m}^{(1)}\right) \otimes \bigotimes_{j+1 \leqslant s \leqslant N} t_{s}^{(0)}\right]_{j k}^{i} \\
& =\left[\bigotimes_{\substack{1 \leq n \leq k \\
n \neq i+1}} t_{n}^{(0)} \otimes t_{k+1}^{(0)}\right. \\
& \quad \otimes \bigotimes_{k+2 \leqslant r \leqslant j} t_{n}^{(0)} \otimes\left(\sigma^{-1} \circ S\right)\left(t_{k+1}^{(1)} \sigma\left(t_{i+1}\right) \prod_{\substack{1 \leq m \leq N \\
m \neq k+1 \\
m \neq i+1}} t_{m}^{(1)}\right) \otimes \underbrace{\otimes}_{j+1 \leqslant s \leqslant N} t_{s}^{(0)}]_{j k}^{i}
\end{aligned}
$$




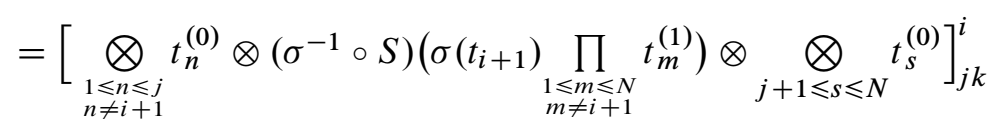

$$
\begin{aligned}
& =\phi_{k}^{i j}\left(\left[\bigotimes_{1 \leqslant n \leqslant N} t_{n}\right]_{i k}^{j}\right) .
\end{aligned}
$$

Our next step is to prove that the assumptions of Lemma 1.1 hold, so that we can take advantage of Birkhoff's Representation Theorem to conclude the freeness of the lattice generated by the ideals $\operatorname{ker} \pi_{i}$.

Lemma 3.3. For all non-empty subsets $I, J \subseteq \underline{N}$

$$
\bigcap_{i \in I} \operatorname{ker} \pi_{i} \supseteq \bigcap_{j \in J} \operatorname{ker} \pi_{j} \quad \text { if and only if } I \subseteq J \text {. }
$$

Proof. The "if"-implication is obvious. For the "only if"-implication, take $0 \neq x \in$ $\mathcal{K}^{\otimes N}$ and, for any non-empty $I \subseteq \underline{N}$, define

$$
x_{I}:=\left(x_{i}\right)_{i \in \underline{N}} \in \bigcap_{i \in I} \operatorname{ker} \pi_{i}, \quad \text { where } x_{i}:= \begin{cases}x & \text { if } i \notin I, \\ 0 & \text { if } i \in I .\end{cases}
$$

Let $I, J \subseteq \underline{N}$ be non-empty, and assume that $I \backslash J$ is non-empty. Then it follows that

$$
x_{J} \in\left(\bigcap_{j \in J} \operatorname{ker} \pi_{j}\right) \backslash\left(\bigcap_{i \in I} \operatorname{ker} \pi_{i}\right) \neq \emptyset .
$$

This means that $\bigcap_{j \in J} \operatorname{ker} \pi_{j} \not \subseteq \bigcap_{i \in I} \operatorname{ker} \pi_{i}$, as desired. Therefore $\bigcap_{i \in I} \operatorname{ker} \pi_{i}$ are all distinct.

Lemma 3.4. The ideals $\bigcap_{i \in I} \operatorname{ker} \pi_{i}$ are all meet (sum) irreducible for arbitrary $\emptyset \neq I \subsetneq \underline{N}$.

Proof. We proceed by contradiction. Suppose that $\bigcap_{i \in I}$ ker $\pi_{i}$ is not meet irreducible for some $\emptyset \neq I \subsetneq \underline{N}$. By Lemma 3.3, $\bigcap_{i \in I} \operatorname{ker} \pi_{i} \neq\{0\}$ because $I \neq \underline{N}$. Hence there exist ideals

$$
a_{\mu}=\sum_{J \in \mathcal{I}_{\mu}} \bigcap_{j \in J} \operatorname{ker} \pi_{j}, \quad \mathcal{g}_{\mu} \subseteq \mathbf{2}^{\underline{N}}, \mu \in\{1,2\},
$$

such that

$$
\bigcap_{i \in I} \operatorname{ker} \pi_{i}=a_{1}+a_{2} \quad \text { and } \quad a_{1}, a_{2} \neq \bigcap_{i \in I} \operatorname{ker} \pi_{i}
$$

In particular, $a_{\mu} \subseteq \bigcap_{i \in I} \operatorname{ker} \pi_{i}, \mu \in\{1,2\}$. On the other hand, if $I \in \mathcal{I}_{\mu}$, then $a_{\mu} \supseteq \bigcap_{i \in I} \operatorname{ker} \pi_{i}$. Hence $a_{\mu}=\bigcap_{i \in I} \operatorname{ker} \pi_{i}$, contrary to our assumption. It follows that, if $\bigcap_{i \in I} \operatorname{ker} \pi_{i}$ is not meet irreducible, then

$$
\bigcap_{i \in I} \operatorname{ker} \pi_{i}=\sum_{J \in \mathcal{I}} \bigcap_{j \in J} \operatorname{ker} \pi_{j}, \quad \text { for some } \mathcal{g} \subseteq \mathbf{2}^{\underline{N}} \backslash\{I\} .
$$


Suppose next that $I \backslash J_{0}$ is non-empty for some $J_{0} \in \mathcal{g}$, and let $k \in I \backslash J_{0}$. Then

$$
\{0\}=\pi_{k}\left(\bigcap_{i \in I} \operatorname{ker} \pi_{i}\right)=\pi_{k}\left(\sum_{J \in \mathcal{L}} \bigcap_{j \in J} \operatorname{ker} \pi_{j}\right) \supseteq \pi_{k}\left(\bigcap_{j \in J_{0}} \operatorname{ker} \pi_{j}\right) .
$$

However, from Lemma 3.3 we see that $\left(\bigcap_{j \in J_{0}} \operatorname{ker} \pi_{j}\right) \backslash \operatorname{ker} \pi_{k}$ is non-empty. Hence $\pi_{k}\left(\bigcap_{j \in J_{0}} \operatorname{ker} \pi_{j}\right)$ is not $\{0\}$, and we have a contradiction. It follows that for all $J_{0} \in \mathcal{G}$ the set $I \backslash J_{0}$ is empty, i.e., $\forall J_{0} \in \mathcal{G}: I \subsetneq J_{0}$.

Finally, let $m \in \underline{N} \backslash I$, and let

$T_{m}^{I}:=t_{1} \otimes \cdots \otimes t_{N}, \quad$ where $0 \neq t_{n} \in \begin{cases}\mathcal{K} \quad \text { if } m<n \in I \text { or } m>n-1 \in I, \\ \mathcal{T} \backslash \mathcal{K} \quad \text { if } m<n \notin I \text { or } m>n-1 \notin I .\end{cases}$

Note that $\pi_{k}^{m}\left(T_{m}^{I}\right)=0$ if and only if $k \in I$. Hence, by Lemma 3.2, there exists $p_{m} \in \pi_{m}^{-1}\left(T_{m}^{I}\right) \cap \bigcap_{i \in I} \operatorname{ker} \pi_{i}$. Next, we define

$$
\sigma_{I}^{m}:=f_{1} \otimes \cdots \otimes f_{N}, \quad \text { where } f_{n}:= \begin{cases}\operatorname{id}_{\mathcal{T}} & \text { if } m<n \in I \text { or } m>n-1 \in I, \\ \sigma & \text { if } m<n \notin I \text { or } m>n-1 \notin I,\end{cases}
$$

so that $\sigma_{I}^{m}\left(\pi_{m}\left(p_{m}\right)\right) \neq 0$. On the other hand, by our assumption (9), and the property that $J_{0} \supsetneq I$ for all $J_{0} \in \mathcal{g}$, we have

$$
0 \neq p_{m} \in \bigcap_{i \in I} \operatorname{ker} \pi_{i} \subseteq \sum_{J \supsetneq I} \bigcap_{j \in J} \operatorname{ker} \pi_{j}
$$

Furthermore, for any $x \in C\left(\mathbb{P}^{N}(\mathcal{T})\right)$

$$
\sigma_{I}^{m}\left(\pi_{m}(x)\right)=0 \quad \text { if } \pi_{k}^{m}\left(\pi_{m}(x)\right)=0 \text { for some } k \notin I .
$$

Now, for any $J \supsetneq I$, we choose $k_{J} \in J \backslash I$, so that

$$
\pi_{k_{J}}^{m}\left(\pi_{m}\left(\bigcap_{j \in J \supsetneq I} \operatorname{ker} \pi_{j}\right)\right) \subseteq \pi_{m}^{k_{J}}\left(\pi_{k_{J}}\left(\operatorname{ker} \pi_{k_{J}}\right)\right)=\{0\} .
$$

Combining this with (11), we obtain $\sigma_{I}^{m}\left(\pi_{m}\left(\bigcap_{j \in J \supsetneq I} \operatorname{ker} \pi_{j}\right)\right)=\{0\}$ for all $J \supsetneq I$. Consequently, $\sigma_{I}^{m}\left(\pi_{m}\left(\sum_{J \supsetneq I} \bigcap_{j \in J} \operatorname{ker} \pi_{j}\right)\right)=\{0\}$, which contradicts (10) and ends the proof.

Summarizing, Lemma 3.3 and Lemma 3.4 combined with Lemma 1.1 yield the main result of this paper:

Theorem 3.5. Let $C\left(\mathbb{P}^{N}(\mathcal{T})\right) \subset \prod_{i=0}^{N} \mathcal{T}^{\otimes N}$ be the $C^{*}$-algebra of the Toeplitz quantum projective space, defined as the limit of diagram (6), and let

$$
\pi_{i}: C\left(\mathbb{P}^{N}(\mathcal{T})\right) \longrightarrow \mathcal{T}^{\otimes N}, \quad i \in \underline{N},
$$

be the family of restrictions of the canonical projections onto the components. Then the family of ideals $\left\{\operatorname{ker} \pi_{i}\right\}_{i \in \underline{N}}$ generates a free distributive lattice. 


\section{Other quantum projective spaces}

Let us first compare our construction of quantum complex projective spaces with the construction coming from quantum groups. Then we complete this comparison by describing other noncommutative versions of complex projective spaces that we found in the literature.

\subsection{Noncommutative projective spaces as homogeneous spaces over quantum}

groups. Complex projective spaces are fundamental examples of compact manifolds without boundary. They can be viewed as the quotient spaces of odd-dimensional spheres divided by an action of the group U(1) of unitary complex numbers. This presentation allows for a noncommutative deformation coming from the world of compact quantum groups via Soibelman-Vaksman spheres. This approach has been widely explored, and recently entered the very heart of noncommutative geometry via the study of Dirac operators on the thus obtained quantum projective spaces [11].

Recall that the $\mathrm{C}^{*}$-algebra $C\left(\mathbb{C} P_{q}^{N}\right)$ of functions on a quantum projective space, as defined by Soibelman and Vaksman [33], is the invariant subalgebra for an action of $\mathrm{U}(1)$ on the $\mathrm{C}^{*}$-algebra of the odd-dimensional quantum sphere $C\left(S_{q}^{2 N+1}\right)$ (cf. [22]). By analyzing the space of characters, we want to show that this $\mathrm{C}^{*}$-algebra is not isomorphic to the $\mathrm{C}^{*}$-algebra $C\left(\mathbb{P}^{N}(\mathcal{T})\right)$ of the Toeplitz quantum projective space proposed in this paper, unless $N=0$. To this end, we first observe that one can easily see from Definition 2.2 that the space of characters on $C\left(\mathbb{P}^{N}(\mathcal{T})\right)$ contains the $N$-torus. On the other hand, since $C\left(\mathbb{C} P_{q}^{N}\right)$ is a graph $\mathrm{C}^{*}$-algebra [17], its space of characters is at most a circle. Hence these $\mathrm{C}^{*}$-algebras can coincide only for $N=0,1$. For $N=0$, they both degenerate to $\mathbb{C}$, and for $N=1$, they are known to be the standard Podleś and mirror quantum spheres, respectively. The latter are non-isomorphic, so that the claim follows.

Better still, one can easily show that the $\mathrm{C}^{*}$-algebras of the quantum-group projective spaces admit only one character. Indeed, these $\mathrm{C}^{*}$-algebras are obtained by iterated extensions by the ideal of compact operators, i.e., for any $N$, there is the short exact sequence of $C^{*}$-algebras [17], eq. 4.11:

$$
0 \longrightarrow \mathcal{K} \longrightarrow C\left(\mathbb{C} P_{q}^{N}\right) \longrightarrow C\left(\mathbb{C} P_{q}^{N-1}\right) \longrightarrow 0
$$

On the other hand, any character on a $\mathrm{C}^{*}$-algebra containing the ideal $\mathcal{K}$ of compact operators must evaluate to 0 on $\mathcal{K}$, as otherwise it would define a proper ideal in $\mathcal{K}$, which is impossible. Therefore, not only any character on $C\left(\mathbb{C} P_{q}^{N-1}\right)$ naturally extends to a character on $C\left(\mathbb{C} P_{q}^{N}\right)$, but also any character on $C\left(\mathbb{C} P_{q}^{N}\right)$ naturally descends to a character on $C\left(\mathbb{C} P_{q}^{N-1}\right)$. Hence the space of characters on $C\left(\mathbb{C} P_{q}^{N}\right)$ coincides with the space of characters on $C\left(\mathbb{C} P_{q}^{N-1}\right)$. Recalling that $C\left(\mathbb{C} P_{q}^{0}\right)=\mathbb{C}$, we conclude the claim. 
4.2. Noncommutative projective schemes. Projective spaces à la Artin-Zhang [3] and Rosenberg [24] are based on Gabriel's Reconstruction Theorem [12], Ch. VI, (cf. [25]) and Serre's Theorem [26], Prop. 7.8 (cf. [13], vol. II, 3.3.5). The former theorem describes how to reconstruct a scheme from its category of quasi-coherent sheaves. The latter establishes how to obtain the category of quasi-coherent sheaves over the projective scheme corresponding to a conical affine scheme. First, one constructs a graded algebra $A$ of polynomials on this conical affine scheme and then, according to Serre's recipe, one divides the category of graded $A$-modules by the subcategory of graded modules that are torsion. Such graded algebras corresponding to projective manifolds have finite global dimension, admit a dualizing module, and their Hilbert series have polynomial growth. All this means that they are, so called, Artin-Schelter regular algebras, or AS-regular algebras in short [1] (cf. [2]). This property makes sense for algebras which are not necessarily commutative, so that we think about noncommutative algebras of this sort as of generalized noncommutative projective manifolds. One important subclass of such well-behaving algebras are Sklyanin algebras [28]. Among other nice properties, they are quadratic Koszul, have finite Gelfand-Kirillov dimension [27], and are Cohen-Macaulay [21]. Another class of AS-regular algebras worth mentioning is the class of hyperbolic rings [24], which are also known as generalized Weyl algebras [4], or as generalized Laurent polynomial rings [10].

4.3. Quantum deformations of Grassmannian and flag varieties. In [30], Taft and Towber develop a direct approach to quantizing the Grassmannians, or more generally, flag varieties. They define a particular deformation of algebras of functions on the classical Grassmannians and flag varieties using an explicit (in terms of generators and relations) construction of affine flag schemes defined by Towber [31, 32]. Their deformation utilizes $q$-determinants [30], Defn. 1.3, (cf. [18], p. 227, and [20], p. 312) used to construct a $q$-deformed version of the exterior product [30, Sect. 2]. This yields a class of algebras known as quantum exterior algebras [5]. These quantum exterior algebras are different from Weyl algebras or Clifford algebras. They provide counterexamples for a number of homological conjectures for finite dimensional algebras, even though they are cohomologically well behaved. See [5], Sect. 1, for more details.

Acknowledgements. This work is part of the EU-project Geometry and symmetry of quantum spaces PIRSES-GA-2008-230836. It was also partially supported by the Polish Government grants N201 177033 (PMH, BZ), 1261/7.PRUE/2009/7 (PMH), 189/6.PRUE/2007/7(PMH), and the Argentinian grant PICT 2006-00836 (AK). Part of this article was finished during a visit of AK at the Max Planck Institute in Bonn. The Institute support and hospitality are gratefully acknowledged. Finally, we are very happy to thank the following people for discussions and advise: Paul F. Baum, Nigel Higson, Masoud Khalkhali, Tomasz Maszczyk, Ralf Meyer and Jan Rudnik. 


\section{References}

[1] M. Artin and W. F. Schelter, Graded algebras of global dimension 3. Adv. in Math. 66 (1987), 171-216. Zbl 0633.16001 MR 917738

[2] M. Artin, J. Tate, and M. Van den Bergh, Some algebras associated to automorphisms of elliptic curves. In The Grothendieck Festschrift, Vol. I, Progr. Math. 86, Birkhäuser, Boston, 1990, 33-85. Zbl 0744.14024 MR 1086882

[3] M. Artin and J. J. Zhang, Noncommutative projective schemes. Adv. Math. 109 (1994), 228-287. Zbl 0833.14002 MR 1304753

[4] V. V. Bavula, Finite-dimensionality of $\operatorname{Ext}^{n}$ and $\operatorname{Tor}_{n}$ of simple modules over a class of algebras. Funktsional. Anal. i Prilozhen. 25 (1991), no. 3, 80-82; English transl. Funct. Anal. Appl. 25 (1991), 229-230. Zbl 0755.17018 MR 1139880

[5] P. A. Bergh, On the Hochschild (co)homology of quantum exterior algebras. Comm. Algebra 35 (2007), 3440-3450. Zbl 1141.16011 MR 2362664

[6] G. Birkhoff, Rings of sets. Duke Math. J. 3 (1937), 443-454. JFM 63.0832.02 Zbl 0017.19403 MR 1546000

[7] G. Birkhoff, Lattice theory. Third edition, Amer. Math. Soc. Colloq. Publ. 25, Amer. Math. Soc., Providence, R.I., 1967. Zbl 0153.02501 MR 0227053

[8] S. Burris and H. P. Sankappanavar, A course in universal algebra. Graduate Texts in Math. 78, Springer-Verlag, New York 1981. Zbl 0478.08001 MR 648287

[9] D. Calow and R. Matthes, Covering and gluing of algebras and differential algebras. J. Geom. Phys. 32 (2000), 364-396. Zbl 0969.46051 MR 1734767

[10] T. Cassidy, P. Goetz, and B. Shelton, Generalized Laurent polynomial rings as quantum projective 3-spaces. J. Algebra 303 (2006), 358-372. Zbl 1104.16023 MR 2253666

[11] F. D'Andrea and L. Dąbrowski, Dirac operators on quantum projective spaces. Comm. Math. Phys. 295 (2010), 731-790. Zbl 1190.58011 MR 2600033

[12] P. Gabriel, Des catégories abéliennes. Bull. Soc. Math. France 90 (1962), 323-448. Zbl 0201.35602 MR 0232821

[13] A. Grothendieck and J. Dieudonné, Eléments de géométrie algébrique: I--IV. Inst. Hautes Études Sci. Publ. Math. 4 (1960), 5-228.

[14] P. M. Hajac, A. Kaygun and B. Zieliński, Finite closed coverings of compact quantum spaces. Preprint 2008. arXiv:0901.0074

[15] P. M. Hajac, U. Krähmer, R. Matthes, and B. Zieliński, Piecewise principal comodule algebras. J. Noncommut. Geom. 5 (2011), 591-614. Zbl 05970193 MR 2838527

[16] P. M. Hajac, R. Matthes, and W. Szymański, Noncommutative index theory for mirror quantum spheres. C. R. Math. Acad. Sci. Paris 343 (2006), 731-736. Zbl 1114.46052 MR 2284701

[17] J. H. Hong and W. Szymański, Quantum spheres and projective spaces as graph algebras. Comm. Math. Phys. 232 (2002), 157-188. Zbl 1015.81029 MR 1942860

[18] C. Kassel, Quantum groups. Graduate Texts in Math. 155, Springer-Verlag, New York 1995. Zbl 0808.17003 MR 1321145 
[19] S. Klimek and A. Lesniewski, A two-parameter quantum deformation of the unit disc. J. Funct. Anal. 115 (1993), 1-23. MR 1228139

[20] A. Klimyk and K. Schmüdgen, Quantum groups and their representations. Texts Monogr. Phys., Springer-Verlag, Berlin 1997. Zbl 0891.17010 MR 1492989

[21] T. Levasseur, Some properties of non-commutative regular graded rings. Glasgow Math. J. 34 (1992), 277-300. Zbl 0824.16032 MR 1181768

[22] U. Meyer, Projective quantum spaces. Lett. Math. Phys. 35 (1995), 91-97. Zbl 0847.17014 MR 1347872

[23] P. Podleś, Quantum spheres. Lett. Math. Phys. 14 (1987), 193-202. Zbl 0634.46054 MR 919322

[24] A. L. Rosenberg, Noncommutative algebraic geometry and representations of quantized algebras. Math. Appl. 330, Kluwer Academic Publishers, Dordrecht 1995. Zbl 0839.16002 MR 1347919

[25] A. L. Rosenberg, The spectrum of abelian categories and reconstruction of schemes. In Rings, Hopf algebras, and Brauer groups (Antwerp/Brussels, 1996), Lecture Notes in Pure and Appl. Math. 197, Dekker, New York 1998, 257-274. Zbl 0898.18005 MR 1615928

[26] J.-P. Serre, Faisceaux algébriques cohérents. Ann. of Math. (2) 61 (1955), 197-278. Zbl 0067.16201 MR 0068874

[27] B. Shelton and C. Tingey, On Koszul algebras and a new construction of Artin-Schelter regular algebras. J. Algebra 241 (2001), 789-798. Zbl 1002.16025 MR 1843325

[28] E. K. Sklyanin, Some algebraic structures connected with the Yang-Baxter equation. Funktsional. Anal. i Prilozhen. 16 (1982), no. 4, 27-34; English transl. Funct. Anal. Appl. 16 (1983), 263-270. Zbl 0536.58007 MR 684124

[29] R. P. Stanley, Enumerative combinatorics. Cambridge Stud. Adv. Math. 49, Cambridge University Press, Cambridge, 1997. Zbl 0889.05001 MR 1442260

[30] E. Taft and J. Towber, Quantum deformation of flag schemes and Grassmann schemes. I. A $q$-deformation of the shape-algebra for GL(n). J. Algebra 142 (1991), 1-36. Zbl 0739.17007 MR 1125202

[31] J. Towber, Two new functors from modules to algebras. J. Algebra 47 (1977), 80-104. Zbl 0358.15033 MR 0469955

[32] J. Towber, Young symmetry, the flag manifold, and representations of GL(n). J. Algebra 61 (1979), 414-462. Zbl 0437.14030 MR 559849

[33] L. L. Vaksman and Y. S. Sorbel'man, Algebra of functions on the quantum group $\mathrm{SU}(n+1)$, and odd-dimensional quantum spheres. Algebra i Analiz 2 (1990), no. 5, 101-120; English transl. Leningr. Math. J. 2 (1991), 1023-1042. Zbl 0751.46048 MR 1086447 
P. M. Hajac, Instytut Matematyczny, Polska Akademia Nauk, ul. Śniadeckich 8, 00-956 Warszawa, Poland, and

Katedra Metod Matematycznych Fizyki, Uniwersytet Warszawski, ul. Hoża 74, 00-682 Warszawa, Poland

E-mail: pmh@impan.pl

A. Kaygun, Department of Mathematics and Computer Science, Bahçeşehir University, Çırağan Cad., Beşiktaş 34353 Istanbul, Turkey

E-mail: atabey.kaygun@bahcesehir.edu.tr

B. Zieliński, Instytut Matematyczny, Polska Akademia Nauk, ul. Śniadeckich 8, 00-956 Warszawa, Poland, and

Department of Theoretical Physics and Computer Science, Faculty of Physics and Applied Informatics, University of Łódź, Pomorska 149/153, 90-236 Łódź, Poland

E-mail: bzielinski@uni.lodz.pl 